\title{
Revista digital
}

Matemática, Educación e Internet

(https://tecdigital.tec.ac.cr/revistamatematica/).

Vol 20, No 2. Marzo - Agosto, 2020

ISSN $1659-0643$

\section{Implementación de la reforma matemática en Costa Rica: una experiencia de capacitación docente en el uso de tecnología | Implementation of the mathematical reform in Costa Rica: a teacher training experience in the use of technology I}

\author{
Evelyn Agüero Calvo \\ evaguero@itcr.ac.cr \\ Escuela de Matemática \\ Instituto Tecnológico de Costa Rica \\ Costa Rica
}

\author{
Adriana Solís Arguedas \\ asolis@itcr.ac.cr \\ Escuela de Matemática \\ Instituto Tecnológico de Costa Rica \\ Costa Rica
}

Recibido: 5 de agosto 2019

Aceptado: 10 de agosto 2019

\section{Resumen.}

Los nuevos programas de matemática para la educación primaria y secundaria de Costa Rica, aprobados en mayo de 2012 por el Consejo Superior de Educación, le dan un papel importante al uso las tecnologías en el aula, ya que uno de sus ejes disciplinares es el uso inteligente y visionario de las tecnologías digitales.

La Escuela de Matemática del Instituto Tecnológico de Costa Rica (ITCR) tiene un compromiso social y no puede abstraerse del entorno, por lo que en el 2018 desarrolló un proyecto de extensión formalmente inscrito ante la Vicerrectoría de Investigación y Extensión, para colaborar con el Ministerio de Educación Pública (MEP) en la implementación de la reforma educativa en matemática.

En el proyecto participaron 72 docentes de primaria provenientes de un total de 40 escuelas públicas de las regiones educativas de Cartago y Turrialba, que asistieron a sesiones de capacitación sobre el uso de tecnología para la enseñanza de la matemática. En este artículo se reportan los resultados de la experiencia, así como aspectos positivos y negativos del proceso de implementación de la reforma educativa manifestados por los docentes con respecto al uso de tecnología.

Además, el proyecto colaboró con el proceso de formación de estudiantes de la carrera Enseñanza de la Matemática con Entornos Tecnológicos (MATEC), que imparte la Escuela de Matemática del ITCR, pues se propició su participación como instructores durante las sesiones de capacitación, lo cual les genera experiencia y los involucra en la atención de necesidades del contexto. 
Palabras clave: Enseñanza de la matemática, capacitación docente, educación primaria, tecnología

Abstract. The new Costa Rican math programs for elementary and high school levels approved in the month of May 2012 by the Council of Higher Education place special emphasis on the use of technology in the classroom. This derives from one of the pillars in math teaching which is based on the use of intelligent and visionary digital technologies.

The Costa Rica Institute of Technology (ITCR) Math School is socially compromised and it cannot abstain itself from contributing to society; therefore, the school developed a community extension project endorsed by the ITCR Deanship of Research and Extension. This project was created to aid the Ministry of Public Education towards the implementation of the math educational reform.

The project's participants included 72 elementary school teachers coming from 40 different public schools in the Cartago and Turrialba areas. These instructors attended training sessions dealing with the use of technology in math teaching. This article shows some findings related to the experience as such as well as positive and negative aspects of the educational reform implementation process from the perspective of the participants.

It is worth mentioning that the project was conducive to the development of math student teachers from the major of Math Teaching in Technological Settings (MATEC) offered by the ITCR School of Math. These math student teachers served as aids and were very actively involved in all the training sessions. They took advantage of this process to gain experience and learn more about how to get involved in addressing the needs of different teaching contexts.

KeyWords: Math teaching, teacher training, elementary education, technology.

\subsection{Introducción}

La sociedad avanza de manera acelerada y es de esperar que la educación, cuyo fin primordial es promover la inserción de las personas en el entorno, se desarrolle en forma paralela a los cambios científicos y tecnológicos enfrentados por el mundo. De acuerdo con Carrillo (2005, p.101) [1] “Las Tecnologías de la Información y la Comunicación (TIC) constituyen un excelente recurso didáctico que es conveniente, cuanto antes, llevar a las aulas para aprovechar las posibilidades que ofrecen para las distintas áreas y niveles educativos". Para este autor, el docente de matemática debe afrontar la labor de introducir las TIC en las aulas con el fin de actualizar los contenidos y el trabajo diario, aprovechando el interés del alumnado hacia estos recursos, pero fundamentalmente para mejorar los procesos de enseñanza y aprendizaje.

Con respecto al uso de las TIC en el aula, Villareal (2012) [12] establece que incluir estas herramientas en los centros educativos debe obedecer a dos premisas básicas, la primera, que todo ciudadano tiene el derecho al acceso de estas tecnologías como parte de un proceso de democratización del conocimiento y la segunda, que en las escuelas, los alumnos se enfrenten a procesos de alfabetización mediante el uso de la tecnología en las actividades que desarrollan en el aula.

Además, Villareal (2012) [12] establece que:

...la cognición incluye herramientas, medios con los cuales se produce el conocimiento y este componente del sujeto epistémico no es auxiliar o suplementario, sino esencial. Tan esencial que ese medio es constitutivo del conocimiento, de suerte que si estuviera ausente el conocimiento construido sería otro. Esta posición epistemológica que coloca a los medios en una situación de coautor en la produc- 
ción del conocimiento brinda una perspectiva que nos permite comprender el papel de la tecnología en la escuela con un carácter potenciador y reorganizador en lugar de supresor del raciocinio (p.79).

El uso de la tecnología en la enseñanza de la matemática ha estado presente en los sistemas educativos de diferentes países desde hace varias décadas, de acuerdo con Lynch (2006) [4], desde los años 80 Australia, Estados Unidos y el Reino Unido promovieron la integración de las computadoras en los currículos escolares. En este sentido, en estudios como los descritos en Santiago, Etxeberria y Lukas (2014) [10], Gamboa (2007) [2] y Salat (2013) [9] se puede constatar que existe un interés vigente en el diseño, implementación y evaluación de actividades para favorecer el aprendizaje de la matemática mediante el uso de computadoras, calculadoras, software para el procesamiento de datos y de software dinámico para geometría.

En el caso de Costa Rica, el Consejo Superior de Educación aprobó en el 2012 una reforma de los programas que habían sido aprobados en 1995, modificando otros que no habían cambiado desde 1964 (Meza, Agüero, Suárez, 2019) [5]. Dicha reforma tiene una importante modificación en la metodología de enseñanza pues establece la resolución de problemas como la metodología principal. De acuerdo con el Ministerio de Educación Pública (MEP, 2012, p.15) [6] los nuevos programas adoptan cinco ejes disciplinares que atraviesan de forma transversal el plan de estudios y fortalecen el currículo:

- La resolución de problemas como estrategia metodológica principal.

- La contextualización activa como un componente pedagógico especial.

- El uso inteligente y visionario de tecnologías digitales.

- La potenciación de actitudes y creencias positivas en torno a las Matemáticas.

- El uso de la historia de las Matemáticas.

Según se indica en los programas (MEP, 2012) [6]: "Las tecnologías pueden ser un poderoso aliado para potenciar el pensamiento matemático. Y es precisamente en la resolución de problemas en entornos reales donde estas pueden aportar sus beneficios de la mejor manera, en contextos de aprendizajes que fortalezcan las habilidades y capacidades matemáticas"(p.37).

Por otro lado, en el Quinto Informe del Estado de la Educación (2015, p.161) [7] se plantea que "en los próximos años un factor crucial para el éxito de la reforma de matemáticas es que el MEP logre fortalecer los procesos de capacitación e integrarlos en el marco de una política de desarrollo profesional de largo plazo, que tenga como norte principal potenciar las habilidades que requieren los docentes para aplicar con éxito los nuevos programas de estudios".

Esta reforma ha estado vigente desde el año 2012 y Meza, Agüero, Suárez (2019) [5] en una investigación realizada durante el 2016 y 2017 determinaron una falta de coherencia entre la dinámica desarrollada en el aula y lo establecido en dichos programas. Estos autores indican que las lecciones observadas se centraron en clases expositivas, trabajo en grupo con apoyo de libros o fotocopias para la resolución de problemas, revisión en la pizarra de los ejercicios y resolución de problemas en formato tradicional con los recursos didácticos tradicionales como libros, fotocopias, pizarra y marcadores, mientras que se encontró una ausencia casi total de tecnología. Por otra parte, destacan cinco elementos que, según los docentes, dificultan la implementación exitosa de los programas: poco tiempo disponible, extensión de los programas, escaso acceso a la tecnología, necesidades de capacitación y la forma de evaluar.

Particularmente, en la Escuela de Matemática del ITCR se ha venido promocionando el uso de la tecnología en la enseñanza de la matemática en el sistema educativo costarricense mediante el proyecto de extensión universitaria denominado: Promoción de la matemática en la educación secundaria (PROMATES) que, según Suárez y Meza (2018) [11], al año 2016, han participado profesores de matemática de 20 colegios públicos costarricenses en procesos de capacitación sobre el uso de software libre para la enseñanza de la matemática.

Implementación de la reforma matemática en Costa Rica: una experiencia de capacitación docente en el uso de tecnología . Evelyn Agüero-Calvo, Adriana Solís-Arguedas

Derechos Reservados (C 2020 Revista digital Matemática, Educación e Internet. ISSN 1659-0643. (https://tecdigital.tec.ac.cr/revistamatematica/) 
Los procesos de capacitación que realiza la Escuela de Matemática a docentes del MEP tienen mucha pertinencia social ya que contribuyen con uno de los objetivos estratégicos que se establecieron en el eje de docencia del Plan Nacional de la Educación Superior Universitaria Estatal 2016-2020 que es “Propiciar el trabajo conjunto entre las universidades y el Ministerio de Educación Pública para incidir en la calidad y pertinencia de la educación nacional"(p.87). Además, encuentran respaldo en la Ley de Promoción del Desarrollo Científico y Tecnológico (Ley No. 7169 del 26 de junio de 1990), que establece como objetivo específico: Fomentar todas las actividades de apoyo al desarrollo científico y tecnológico sustantivo; los estudios de posgrado y la capacitación de recursos humanos, así como el mejoramiento de la enseñanza de las ciencias, las matemáticas y la educación técnica, lo mismo que la documentación e información científica y tecnológica.

En este sentido, los procesos de capacitación docente contribuyen a la misión del ITCR, ya que el Modelo Académico (2003) [3], entre otras cosas, indica lo siguiente:

El Instituto Tecnológico de Costa Rica no puede abstraerse del entorno; por el contrario, debe participar de su quehacer a toda la sociedad. Es por ello que asume un compromiso con el mejoramiento de los otros niveles educativos de la sociedad con el fin de garantizar el derecho al acceso y la permanencia exitosa de todas las personas con potencial para ingresar a instituciones de excelencia académica con las características del Instituto Tecnológico de Costa Rica (p.3).

La importancia de la capacitación de los educadores en servicio es resaltada por el Informe del Estado de la Educación (2008, p. 162) [8] de la siguiente manera: "las acciones de capacitación no son marginales o adicionales a la construcción del conocimiento que requiere el educador, son cruciales", por lo que los esfuerzos de la Escuela de Matemática del ITCR en este aspecto han establecido una línea de colaboración con el MEP, con los docentes en formación y en servicio, y con la sociedad costarricense.

Además, incluir docentes en formación como los estudiantes de la carrera MATEC en proyectos de capacitación es fundamental para los procesos de acreditación ante el Sistema Nacional de Acreditación de la Educación Superior (SINAES), ya que se contribuye a contar con políticas y acciones concretas que favorezcan la participación de los estudiantes de la carrera en la atención de necesidades del contexto; demostrar que se aprovecha el entorno para experiencias prácticas del estudiantado según los requerimientos de la carrera; que el plan de estudios y las estrategias didácticas estimulen, en los estudiantes, su capacidad de aprender, incluyendo componentes orientados a desarrollar, en ellos, pensamientos, principios y prácticas científicas rigurosas relevantes para su disciplina y a ofrecer al estudiantado actividades extracurriculares que complementan el plan de estudios. De esta manera, mediante este tipo de proyectos se abre un espacio a estudiantes de la carrera MATEC en el que pueden poner en práctica los conocimientos que adquieren sobre el uso de tecnología para enseñar, en su papel como instructores del proyecto.

\subsection{Metodología}

El proyecto se desarrolló bajo un enfoque de investigación participativa en el cual los docentes recibieron capacitaciones en el uso de tecnología para los procesos educativos y a la vez aportaron resultados de utilidad para mejorar la implementación de la reforma educativa. Cabe destacar que todas las aplicaciones seleccionadas para el proyecto son de uso libre, lo cual implica que las escuelas no deben incurrir en gastos adicionales para que los docentes hagan uso de las mismas en sus clases.

\section{Acceso al campo}

Para facilitar la participación de los docentes se coordinó con los asesores regionales de las direcciones educativas de Cartago y Turrialba para que realizaran una convocatoria de carácter oficial a las capacitaciones y seleccionaran los docentes. De esta manera, los participantes contaban con el permiso de sus respectivos superiores para desplazarse a las instalaciones del ITCR y recibir las capacitaciones.

Implementación de la reforma matemática en Costa Rica: una experiencia de capacitación docente en el uso de tecnología . Evelyn Agüero-Calvo, Adriana Solís-Arguedas

Derechos Reservados (C) 2020 Revista digital Matemática, Educación e Internet. ISSN 1659-0643. (https://tecdigital.tec.ac.cr/revistamatematica/) 


\section{Participantes}

En el proyecto participaron 72 docentes de primaria, provenientes de un total de 40 escuelas de las regiones educativas de Cartago y Turrialba (una escuela geográficamente está en la provincia de Limón pero pertenece a la región educativa de Turrialba). En la Tabla 1.1 se muestra la distribución por cantones:

Tabla 1.1: Distribución por cantones de las escuelas participantes

\begin{tabular}{|l|l|l|c|}
\hline Provincia & Cantón & Distritos & Cantidad de escuelas \\
\hline Cartago & Cartago & Cartago, Guadalupe, Occidental y Oriental & 7 \\
\hline Limón & Turrialba & $\begin{array}{l}\text { Chirripó, La Isabel, La Suiza, Pavones, Santa Cruz, } \\
\text { Santa Rosa, Santa Teresita, Turrialba, Tres Equis y } \\
\text { Tuis }\end{array}$ & 29 \\
\hline & Jiménez & $\begin{array}{l}\text { Pejibaye y Juan Viñas } \\
\text { Valle La Estrella }\end{array}$ & 3 \\
\hline
\end{tabular}

Fuente: Elaboración propia

\section{Estrategia para la recolección de datos}

Como estrategia fundamental para la recolecta de datos se desarrollaron sesiones presenciales y virtuales durante el año 2018. Cada mes, desde abril hasta noviembre, se capacitó un grupo diferente de docentes para un total de 7 grupos. Durante los meses de octubre y noviembre, la cantidad de docentes convocados disminuyó, pues algunos centros educativos estaban participando de la huelga nacional de educadores, pero eso no afectó el desarrollo normal de los talleres.

Cada grupo de docentes recibió dos sesiones de capacitación presencial y dos sesiones de capacitación virtual. Después de cada sesión presencial, el docente debía poner en práctica lo aprendido con sus estudiantes, desarrollando actividades en el aula mediante el uso de las aplicaciones educativas.

Para favorecer la comunicación entre los docentes e instructores y el envío y entrega de tareas, se utilizó la plataforma educativa Edmodo. Esta plataforma permite fácilmente crear grupos, asignar tareas, programar su envío y gestionar el proceso, además le permite al docente llevar un control sobre el avance de cada estudiante.

Durante los talleres presenciales los docentes participantes aprendieron a usar las siguientes aplicaciones educativas:

1. Edmodo: Plataforma educativa que conecta profesores, estudiantes y padres de familia. Esta fue la plataforma que se utilizó para gestionar dudas de los docentes y la entrega de las evidencias del uso de las aplicaciones en el aula.

2. Plickers: Es una herramienta que permite recopilar datos de evaluaciones en tiempo real, sin que los estudiantes tengan ningún tipo de dispositivo móvil.

3. Generador de códigos QR: Un código QR es un sistema para almacenar información, la cual es fácilmente accesible desde un dispositivo con cámara y un lector de código QR instalado.

4. Quizziz: Es una plataforma que permite hacer evaluaciones en el aula, a manera de juego. Tiene elementos como avatares y memes que hacen que sea muy divertida y lúdica. Además, tiene la opción de que los estudiantes realicen las evaluaciones como tarea.

5. Educaplay: Plataforma que permite crear y compartir actividades multimedia educativas como crucigramas, sopas de letras, dictados, mapas interactivos, videoquices y otros.

6. Google Sites: Es una herramienta para la creación de páginas web. Esta aplicación permite crear un sitio web de una forma tan sencilla como editar un documento.

7. Quizlet: es una aplicación con la cual se pueden realizar fichas interactivas, las cuales pueden realizar tanto los estudiantes como los profesores.

Implementación de la reforma matemática en Costa Rica: una experiencia de capacitación docente en el uso de tecnología . Evelyn Agüero-Calvo, 
Al inicio de la capacitación los docentes participantes respondieron un instrumento para conocer su percepción con respecto al uso de la tecnología para enseñar matemática y al final de la capacitación, otro conocido como Evaluación PIM (Positivo, Interesante, Mejora) para conocer sobre aspectos positivos, interesantes y por mejorar en los talleres.

Los datos recabados durante las sesiones de capacitación son de naturaleza cualitativa, por lo que el análisis de los datos se hizo categorizándolos de manera que se agruparan conceptualmente las unidades que son cubiertas por un mismo tema. Las categorías de análisis se indican en la Tabla 1.2:

Tabla 1.2: Categorías de análisis

\begin{tabular}{|l|l|}
\hline Tema & Categorías \\
Aspectos positivos & $\begin{array}{l}\text { Sobre el software utilizado } \\
\text { Sobre los instructores } \\
\text { Sobre el lugar de capacitación } \\
\text { Sobre el equipo utilizado } \\
\text { Sobre lo aprendido }\end{array}$ \\
\hline Aspectos interesantes & $\begin{array}{l}\text { Sobre utilización de lo aprendido en clases } \\
\text { Sobre emociones mostradas por los niños }\end{array}$ \\
\hline Aspectos por mejorar & $\begin{array}{l}\text { Sobre la duración } \\
\text { Sobre el equipo y recursos }\end{array}$ \\
\hline Dificultades en el aula & $\begin{array}{l}\text { Sobre el equipo y recursos } \\
\text { Sobre las necesidades de capacitación docente }\end{array}$ \\
\hline
\end{tabular}

Fuente: Elaboración propia

\subsection{Resultados}

\section{Características de los participantes}

En las Tablas 1.3 y 1.4 se muestran los resultados de los análisis descriptivos de la distribución de los participantes por sexo y por años de experiencia:

Tabla 1.3: Distribución por sexo

\begin{tabular}{|l|c|c|}
\hline Sexo & Frecuencia absoluta & Frecuencia relativa \\
\hline Hombres & 7 & 9.7 \\
\hline Mujeres & 65 & 90.3 \\
\hline Total & 72 & 100 \\
\hline
\end{tabular}

Fuente: Elaboración propia

En la Tabla 1.5 se muestra la clasificación con respecto al nivel de percepción de los docentes hacia el uso de la tecnología para la enseñanza (un docente decidió no responder):

Cabe resaltar que el nivel de percepción del uso de la tecnología para la enseñanza era alto o muy alto para casi $55 \%$ de los participantes lo cual evidencia una buena disposición hacia el tema y, además, 62,5\% de los participantes tiene más de 15 años de laborar en la docencia.

\section{Aspectos positivos}

Con respecto a los aspectos positivos de la capacitación manifestados por los docentes, se identificaron y clasificaron por categorías como se muestra a continuación: 
Tabla 1.4: Distribución por años de experiencia

\begin{tabular}{|l|c|c|}
\hline Años de experiencia & Frecuencia absoluta & Frecuencia relativa \\
\hline Menos de 5 años & 9 & 12.5 \\
\hline Entre 5 y 10 años & 12 & 16.67 \\
\hline Entre 10 y 15 años & 6 & 8.33 \\
\hline Más de 15 años & 45 & 62.5 \\
\hline Total & 72 & 100 \\
\hline
\end{tabular}

Fuente: Elaboración propia

Tabla 1.5: Nivel de Percepción

\begin{tabular}{|l|c|c|}
\hline Nivel & Frecuencia absoluta & Frecuencia relativa \\
\hline Muy bajo & 1 & 1.41 \\
\hline Bajo & 4 & 5.63 \\
\hline Medio & 27 & 38.03 \\
\hline Alto & 28 & 39.43 \\
\hline Muy alto & 11 & 15.50 \\
\hline Total & 71 & 100 \\
\hline
\end{tabular}

Fuente: Elaboración propia

- Sobre el software utilizado: excelente, útil, novedoso, utilizable en clases, interesante, interactivo, dinámico, fácil de aplicar.

- Sobre los instructores: excelente atención, manejo adecuado del tiempo, respuesta oportuna a las dudas, dominio de los temas, buen acompañamiento, simpáticos, amables, tolerantes, calidez en el trato, buenas explicaciones, disposición, profesionalismo, paciencia, buena comunicación, excelente actitud.

- Sobre el lugar de capacitación: bonito, buen clima, buen espacio, fácil acceso.

- Sobre el equipo utilizado: adecuado, disponible, funcional, con acceso a internet.

- Sobre lo aprendido: muy enriquecedor, transmitible a los estudiantes, permite crear actividades lúdicas, permite hacer clases más participativas y dinámicas, nuevas modalidades de uso de tecnología, nuevas formas de usar celulares para los niños, viable.

\section{Aspectos interesantes}

Con respecto a los aspectos interesantes de la capacitación manifestados por los docentes, se identificaron y clasificaron por categorías como se muestra a continuación:

- Sobre utilización de lo aprendido en clases: innovador, útil en cualquier área, fácil crear página web, docentes motivados, permite atraer atención de los estudiantes, integración del juego en la enseñanza, actualización docente, fácil de usar hasta en personas no acostumbradas a usar tecnología, se aprende mucho, materia dinámica, uso en todas las edades y materias, hacer cosas que antes pensaba eran inalcanzables, adaptación a cualquier nivel y materia, uso de nuevas técnicas, aplicaciones bonitas y divertidas para la enseñanza, aprendizaje de programas que no se tenía idea que existían.

- Sobre emociones mostradas por los niños: agrado, interés, diversión, motivación, disfrute, fascinación.

\section{Aspectos por mejorar}

Con respecto a los aspectos por mejorar de la capacitación manifestados por los docentes, se identificaron y clasificaron por categorías como se muestra a continuación: 
- Sobre la duración: más horas de capacitación presencial, más tiempo para aprender cada aplicación educativa, más horas de práctica, más tiempo para aclarar dudas, dar más capacitaciones de este tipo.

- Sobre el equipo y recursos: mayor velocidad de internet.

\section{Dificultades en el aula}

Con respecto a las dificultades de uso de tecnología en los salones de clase manifestadas por los docentes, se identificaron y clasificaron por categorías como se muestra a continuación:

- Sobre el equipo y recursos: no hay internet en todos los centros educativos, poco equipo disponible para uso de los estudiantes, internet solo para uso administrativo, el laboratorio no se puede usar para otras materias.

- Sobre las necesidades de capacitación docente: los docentes no están familiarizados con las nuevas tecnologías, no se sabe usar la tecnología para dar clases, poco conocimiento de aplicaciones para usar en clases, dar capacitaciones a inicios de año, capacitaciones más seguidas, capacitar a todo el personal de la escuela, capacitación constante.

\subsection{Conclusiones}

Los docentes participantes de este proyecto tuvieron la oportunidad de conocer y utilizar diferentes aplicaciones gratuitas con las que pueden desarrollar actividades educativas lúdicas para compartir fácilmente con sus estudiantes. La experiencia adquirida por ellos les generó diversas opiniones positivas e interesantes tales como que las aplicaciones son útiles, novedosas, interactivas y que lo aprendido es muy enriquecedor, fácil de transmitir a los estudiantes y que les permite hacer clases más divertidas, dinámicas y participativas. Esto es resultado positivo ya que $70 \%$ de los docentes participantes tienen más de 10 años de experiencia docente y no necesariamente prefieren métodos de enseñanza tradicionales.

Se suele creer que la reforma educativa que entró en vigencia en el 2012 genera cierta resistencia hacia el uso de la tecnología en educación pero, mediante este proyecto, se encuentra que $55 \%$ de los docentes participantes tienen un nivel de percepción alto o muy alto hacia el uso de la tecnología para la enseñanza y, se puede concluir, que con capacitaciones y recursos adecuados pueden llegar a hacer un uso efectivo y correcto de la tecnología en el aula.

Adicionalmente, los resultados de esta investigación apoyan la notoria necesidad de mejorar los equipos tecnológicos disponibles en los centros educativos costarricenses para uso de los estudiantes, de manera que el docente pueda contar con los recursos adecuados y funcionales para cumplir con el programa del MEP que les pide el uso inteligente y visionario de las tecnologías digitales. El tema del acceso a internet para los estudiantes dentro del centro educativo era un tema recurrente manifestado por los docentes cuando se les preguntaba acerca de las dificultades de usar la tecnología en el aula: que no haya en el centro educativo o que sea solo de uso administrativo es una limitante importante para cumplir con lo establecido en los programas.

Las necesidades de formación son continuas ante una sociedad cambiante, y los docentes necesitan urgentemente tener acceso a aplicaciones novedosas, útiles e interactivas que pueden utilizar en los diferentes niveles y materias; ellos mismos manifiestan no estar familiarizados con la tecnología, ni con las diferentes aplicaciones, ni tampoco saben cómo usarlas, por lo que otro tema recurrente al mencionar dificultades es que la capacitación continua con personas especializadas en el tema es fundamental para el desarrollo de su labor educativa.

Otro aspecto relevante es el fomento de la participación de estudiantes de la carrera MATEC como instructores de aplicaciones educativas, lo cual permite contribuir a su desarrollo profesional como futuros educadores haciéndo- 
los partícipes del contexto y de la realidad nacional.

Mediante este tipo de proyectos de capacitación a docentes de primaria se colabora con el MEP al incidir en la calidad y pertinencia de la educación costarricense, teniendo el ITCR un impacto social fundamental, ya que las universidades no pueden abstraerse del entorno que les rodea y deben ser entes activos en el compromiso del mejoramiento de los otros niveles educativos de la sociedad.

Agradecimiento. Se agradece el apoyo a la Vicerrectoría de Investigación y Extensión del ITCR, a las Direcciones Regionales de Educación de Cartago y Turrialba y a los docentes participantes.

\section{Bibliografía}

[1] Carrillo, A. (2005). Matemáticas a través de las tecnologías de la información y la comunicación. Revista Iberoamericana de Educación Matemática, (3), pp. 101-102.

[2] Gamboa, R. (2007). Cuadernos de investigación y formación en educación matemática, 2(3), pp. 11-44.

[3] Instituto Tecnológico de Costa Rica. (2003). Modelo Académico del Instituto Tecnológico de Costa Rica. Recuperado de https://www.tec.ac.cr/sites/default/files/media/doc/modelo_academico_ del_itcr.pdf

[4] Lynch, J. (2006). Assessing efects of technology usage on mathematics learning. Mathematics Education Research Journal, 18(3), pp. 29-43.

[5] Meza, L.G; Agüero, E. y Suárez, Z. (2019). Reforma de la educación matemática en Costa Rica: evaluación de avance de la implementación en la educación secundaria. Revista digital Matemática, Educación e Internet Vol 19, No 2. Marzo Agosto, 2019

[6] Ministerio de Educación Pública (2012). Programas de estudio de matemáticas. I y II Ciclo de la Educación Primaria. III Ciclo de Educación General Básica y Educación Diversificada. San José, Costa Rica.

[7] Programa Estado de la Nación (2015). Quinto Informe Estado de la Educación. San José, Costa Rica. Recuperado de http://www.estadonacion.or.cr/educacion2015/

[8] Programa Estado de la Nación. (2008). Segundo Informe Estado de la Educación. San José, Costa Rica.

[9] Salat, R. (2013) La enseñanza de las matemáticas y la tecnología. Innovación Educativa, 13(62), pp. 61-74.

[10] Santiago, K.; Etxeberria; J. y Lukas,J. (2014). Aprendizaje de las matemáticas mediante el ordenador en Educación Primaria, Revista de Investigacion Educativa, 32(1), pp. 91-109.

[11] Suárez, Z. y Meza, L. (2018). PROMATES: Promoción de la matemática en la educación secundaria. Un proyecto de extensión de la Escuela de Matemática del TEC. Revista Digital Matemática, Educación e Internet, 18(2), pp. 1-11.

[12] Villareal, M. (2012). Tecnologías y educación matemática: necesidad de nuevos abordajes para la enseñanza. VEsC, 3(5), pp. 73-94. Recuperado de https://revistas.unc.edu.ar/index.php/vesc/ article/view/3014

Implementación de la reforma matemática en Costa Rica: una experiencia de capacitación docente en el uso de tecnología . Evelyn Agüero-Calvo, Adriana Solís-Arguedas

Derechos Reservados (C 2020 Revista digital Matemática, Educación e Internet. ISSN 1659-0643. (https://tecdigital.tec.ac.cr/revistamatematica/) 